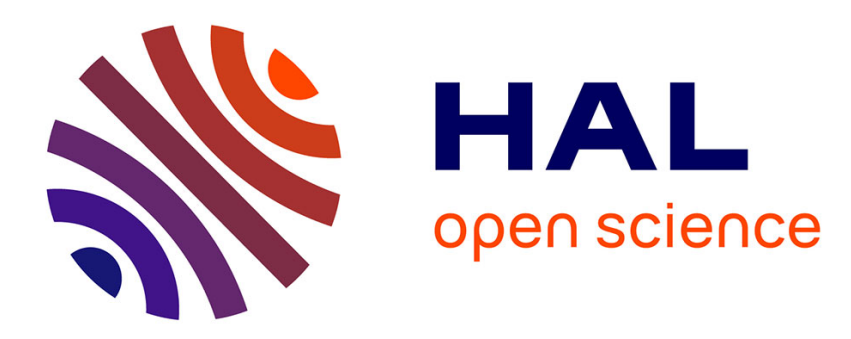

\title{
Asymptotic behavior for a class of the renewal nonlinear equation with diffusion
}

\author{
Philippe Michel, Tarik Touaoula
}

\section{To cite this version:}

Philippe Michel, Tarik Touaoula. Asymptotic behavior for a class of the renewal nonlinear equation with diffusion. Mathematical Methods in the Applied Sciences, 2012, 36 (3), pp.323-335. 10.1002/mma.2591 . hal-00785171

\section{HAL Id: hal-00785171 https://hal.science/hal-00785171}

Submitted on 9 Mar 2017

HAL is a multi-disciplinary open access archive for the deposit and dissemination of scientific research documents, whether they are published or not. The documents may come from teaching and research institutions in France or abroad, or from public or private research centers.
L'archive ouverte pluridisciplinaire HAL, est destinée au dépôt et à la diffusion de documents scientifiques de niveau recherche, publiés ou non, émanant des établissements d'enseignement et de recherche français ou étrangers, des laboratoires publics ou privés. 


\title{
ASYMPTOTIC BEHAVIOR FOR A CLASS OF THE RENEWAL NONLINEAR EQUATION WITH DIFFUSION.
}

\author{
PHILIPPE MICHEL AND TARIK MOHAMED TOUAOULA
}

\begin{abstract}
In this paper we consider nonlinear age structured equation with diffusion under nonlocal boundary condition and nonnegative initial data. More precisely, we prove that under some assumptions on the nonlinear term in a model of McKendrick-Von Foerster with diffusion in age, solutions exist and converge (long time convergence) towards a stationary solution. In the first part, we use classical analysis tools to prove the existence, uniqueness and the positivity of the solution. In the second part, using comparison principle, we prove the convergence of this solution towards the stationary solution.
\end{abstract}

\section{INTRODUCTION}

In the study of population of cells, animals or humans, one of the most used model is the McKendrick-Von Foerster model said also renewal model, where the density of population $n(t, x)$ at time $t$ and age $x$, is described by the master equation

$$
\left\{\begin{array}{l}
n_{t}(t, x)+n_{x}(t, x)+d(x) n(t, x)=0, \quad t \geq 0, \quad x \geq 0 \\
n(t, 0)=\int_{0}^{\infty} B(x) n(t, x) d x, \text { and } n(0, x)=n_{0}(x)
\end{array}\right.
$$

where $B \geq 0$ is the birth rate, $d \geq 0$ is the death rate. It's well known that the long time asymptotic is described by the first eigenvalue $\lambda$ and positive engenvector $N$ of the stationary problem of (1.1). More precisely, for a large time, $n \sim C s t N e^{\lambda t}$, see for instance [10], [11], [18] (using General Relative Entropy method) and (for instance) [7, 8] (using semigroup method). This of course does not take in account the use of resources. Indeed, the population growth is not limited in time when the Malthusian growth rate (eigenvalue $\lambda$ ) is strictly positive. Nevertheless resources are limited and so we expect that there is a limitation of the size of the population (an "equilibrium" between the quantity of resources and their use). On the other hand, we notice that the population goes to the extinction when the Malthusian growth rate is strictly negative. Nevertheless, in this case there is no limitation of the resource and the extinction cannot be explained by the lack of resources. In order to take in account the consumption of nutrient, we can for instance change the linear birth term in (1.1) by a nonlinear birth term (see for instance $[5,6,9,21]$ ) :

$$
\left\{\begin{array}{l}
n_{t}(t, x)+n_{x}(t, x)+d(x) n(t, x)=0, \quad t \geq 0, \quad x \geq 0 \\
n(t, 0)=f\left(\int_{0}^{\infty} B(x) n(t, x) d x\right), \text { and } n(0, x)=n_{0}(x) .
\end{array}\right.
$$

In [2], [3], the authors propose to define the 'biological age' according to the DNA content and diffusion accounts for its variability. Therefore considering that the variable $x$ is a biological age

Key words and phrases. McKendrick-Von Foerster model, iterative method, Asymptotic analysis. 2000 Mathematics Subject Classification: 35B40, 35F10, 92D25.

The authors are partially supported by projects A-8174-07, AECI, Spain. 
and can vary according to certain proteins causing degradation or recovery caused by external factors, the density of the population satisfies the master equation

$$
\left\{\begin{array}{l}
n_{t}(t, x)-n_{x x}(t, x)+(g(x) n(t, x))_{x}+d(x) n(t, x)=0 \quad t \geq 0, \quad x \geq 0 \\
g(0) n(t, 0)-n_{x}(t, 0)=f\left(\int_{0}^{\infty} B(x) n(t, x) d x\right), \quad \text { and } n(0, x)=n_{0}(x) \in L_{+}^{1}\left(\mathbb{R}^{+}\right),
\end{array}\right.
$$

where $B \geq 0$ is the birth rate, $d \geq 0$ is the death rate and the diffusion term modelizes the variability evolution of the 'biological age' $x$. We notice that the problem (1.2) arises in many other applications, see for instance [4],[13], [14], [20] and the references therein. Recently we have treated the linear case of this renewal model with diffusion (see [1]), we proved the existence, uniqueness and positivity of the solution, and we showed that for a large time, $n \sim C s t N e^{\lambda t}$ (with the decay estimate of the solution). Nevertheless it does not take into account the consumption of resources. In the system (1.2) we overcome this, and consider the consumption of the resources like nutrients, by introducing nonlinearity in the birth term. In general this term may limit the possible extra growth of the population. In the system (1.2), we notice that the nonlinear form of the recruitment term only takes in accounts newborns. This means that, in a population modelized by system (1.2), "giving birth" and "newborns" need more resources and may limit growth of the population.

\section{Assumptions And main Results}

In this section we give the main assumptions of this work and state the main results of the paper We suppose that $B$ and $d$ are nonnegative continuous functions and satisfy

$$
0<B_{m} \leq B(x) \leq B_{M} \text { and } 0<d_{m} \leq d(x) \leq d_{M}
$$

The nonlinear growth function $f$ is smooth (for instance $C^{1}(] 0, \infty[)$ ), nondecreasing and verifies the condition

$$
f(x) \leq \alpha x+\gamma
$$

with $\gamma$ positive and $\alpha \in[0, A)$ with a constant $A$ to be chosen later.

$$
\left.\exists s_{0}>0: \forall s^{\prime} \in\right] 0, s_{0}\left[f\left(s^{\prime}\right) / s^{\prime}>d_{M} / B_{m}\right.
$$

and

$$
\left.\exists s_{1}>0: \forall s^{\prime} \in\right] s_{1}, \infty\left[f\left(s^{\prime}\right) / s^{\prime}<d_{m} / B_{M} .\right.
$$

The growth rate $g$ is a $C^{1}$ positive function satisfying,

$$
\begin{gathered}
0<g_{m} \leq g(x) \leq g_{M} \\
\int_{0}^{\infty} e^{-G(x)} d x<\infty
\end{gathered}
$$

where $G(x):=\int_{0}^{x} g(s) d s$, and there exists a positive constant $\varepsilon$ such that

$$
d(x)+g^{\prime}(x) \geq \varepsilon
$$


Remark 2.1. We notice that the condition (2.3) (resp. (2.4)) avoid the extinction (resp. the unlimited growth) of the population which cannot be explained by the lack of resources. Indeed, under the existence of solutions to (1.2) (proved in section 3), we have

$$
\frac{d}{d t} \int_{0}^{\infty} n(t, x) d x \geq f\left(B_{m} \int_{0}^{\infty} n(t, x) d x\right)-d_{M} \int_{0}^{\infty} n(t, x) d x
$$

and the condition (2.3) implies that $\int_{0}^{\infty} n(t, x) d x \geq \min \left(\int_{0}^{\infty} n_{0}(x) d x, s_{0}\right)$ for all time $t \geq 0$. This means that the trivial solution is not stable. For instance, the condition (2.3) is satisfied for functions which behave as $f(t) \sim_{t \rightarrow 0} C t^{\alpha}$ with $\alpha<1$ and $C>0$ or $\alpha=1$ and $C>d_{M} / B_{m}$.

We will prove the following result, which concern the existence and uniqueness of the positive solution.

Theorem 2.2. Under assumptions (2.1)-(2.6) and for all positive initial datum $n_{0} \in L^{1}\left(\mathbb{R}^{+}, e^{-G(x)} d x\right)$, the problem (1.2) has a unique positive solution $n \in C\left([0, T], L^{1}\left(\mathbb{R}^{+}, e^{-G(x)} d x\right)\right)$.

The following theorem concern the asymptotic behavior of the solution $n$ to problem (1.2). To be more precise, let $N$ be solution of the following stationary problem

$$
\left\{\begin{array}{l}
-N^{\prime \prime}(x)+(g N)^{\prime}(x)+d(x) N(x)=0, \quad x \geq 0, \\
g(0) N(0)-N^{\prime}(0)=f\left(\int_{0}^{\infty} B(x) N(x) d x\right), \\
\int_{0}^{\infty} N(x) d x<\infty, \text { and } N \geq 0 .
\end{array}\right.
$$

Then we have,

Theorem 2.3. Assume that (2.1)-(2.7) hold. Then for all positive initial datum $n_{0} \in L^{1}\left(\mathbb{R}^{+}, e^{-G(x)} d x\right)$, with $n_{0}(x) \leq C N(x)$, the solution $n$ to (1.2) satisfies

$$
\underline{N}(x) \leq \liminf _{t \rightarrow \infty} n(t, x) \leq \limsup _{t \rightarrow \infty} n(t, x) \leq \bar{N}(x),
$$

where $\bar{N}$ (resp. $\underline{N}$ ) is the maximal (resp. minimal) non trivial solution to the stationary problem (2.8). In addition if the stationary problem (2.8) has an unique non trivial solution $N$ then we have the convergence of $n(t, x)$ to $N(x)$.

The paper is organized as follows. In section 3 we prove the existence, uniqueness and positivity of solutions to the problems (1.2). Section 4 is devoted to prove the convergence of solutions towards the solution of the stationary problem $N$. In the section 5 we prove a blow up result and an extinction result under some assumptions on the growth function $f$. Finally, the paper is supplemented by the numerical simulation, and discuss on the model and the theoretical results.

\section{Existence RESUlts}

In this section we will prove the existence, uniqueness and positivity of the solution to the nonlinear partial differential equation (1.2). The following theorem is devoted to prove the existence, uniqueness and positivity of the solution to problem (1.2) in a regularized space. More precisely,

Theorem 3.1. Under assumptions (2.1)-(2.6) and for all positive initial datum $n_{0} \in L^{1}\left(\mathbb{R}^{+}\right) \cap$ $L^{2}\left(\mathbb{R}^{+}\right)$, there is a unique positive solution $n$ to problem $(1.2)$ that belongs to $\mathcal{C}\left([0, \infty) ; L^{2}\left(\mathbb{R}^{+}\right)\right) \cap$ $L^{1}\left((0, T) \times \mathbb{R}^{+}\right) \cap L^{2}\left(0, T ; W^{1,2}\left(\mathbb{R}^{+}\right)\right)$for all $T>0$. 
We begin by stating the comparison principle lemma which is very useful for the rest of the paper. We consider the following problem

$$
\left\{\begin{array}{l}
n_{t}(t, x)-n_{x x}(t, x)+(g(x) n(t, x))_{x}+d(x) n(t, x)=0, \quad t \geq 0, \quad x \geq 0 \\
g(0) n(t, 0)-n_{x}(t, 0)=\alpha \int_{0}^{\infty} B(x) n(t, x) d x+\gamma, \quad \text { and } n(0, x)=n_{0}(x) \in L^{1}\left(\mathbb{R}^{+}\right),
\end{array}\right.
$$

then we have

Lemma 3.2. Let $v$ and $u$ be a nonnegative supersolution and a nonnegative subsolution of problem (3.1), respectively. If $v(0, x) \geq u(0, x) \geq 0$ then $v(t, x) \geq u(t, x) \geq 0$.

Proof. Let $\phi \in L^{2}\left((0, T) ; W^{1,2}\left(\mathbb{R}^{+}\right)\right)$be a solution to a following problem

$$
\left\{\begin{array}{l}
\phi_{\tau}(\tau, x)+\phi_{x x}(\tau, x)+g(x) \phi_{x}(\tau, x)-d(x) \phi(\tau, x)=0, \quad 0<\tau<t, \quad x \geq 0, \\
\phi_{x}(\tau, 0)=0, \text { and } \phi(t, x)=\psi(t, x),
\end{array}\right.
$$

where $\psi(t, x)=1_{\{u-v>0\}}$. Is not difficult to see that the problem (3.2) has a positive solution. By setting $w=u-v$, we have

$$
\left\{\begin{array}{l}
w_{t}(t, x)-w_{x x}(t, x)+(g(x) w(t, x))_{x}+d(x) w(t, x) \leq 0, \quad t \geq 0, \quad x \geq 0 \\
g(0) w(t, 0)-w_{x}(t, 0) \leq \int_{0}^{\infty} B(x) w^{+}(t, x) d x, \text { and } w(0, x) \leq 0 .
\end{array}\right.
$$

If we multiply the first inequality in (3.3) by $\phi$ and integrate over $(0, t) \times \mathbb{R}^{+}$we get

$$
\int_{0}^{\infty} w(t, x) \phi(t, x) d x \leq \int_{0}^{t} \int_{0}^{\infty} \phi(\tau, 0) B(x) w(\tau, x) d x d \tau
$$

Hence from (3.4) we obtain

$$
\int_{0}^{\infty} w^{+}(t, x) d x \leq M \int_{0}^{t} \int_{0}^{\infty} w^{+}(\tau, x) d x d \tau
$$

Applying Gronwall's inequality, the conclusion of this theorem follows.

The existence, uniqueness and positiveness of the solution to problem (3.1) are stated in the following Lemma,

Lemma 3.3. Let $n_{0} \in L^{1}\left(\mathbb{R}^{+}\right) \cap L^{2}\left(\mathbb{R}^{+}\right)$, then the problem (3.1) has a unique positive solution in $L^{1}\left((0, T) \times \mathbb{R}^{+}\right) \bigcap L^{2}\left((0, T) ; W^{1,2}\left(\mathbb{R}^{+}\right)\right)$.

Proof. The proof of this lemma is almost similar as in [1], for the sake of completeness we done it.

To prove the existence result we will argue by approximation, namely we consider the case of bounded domain $[0, R]$ and then we pass to the limit in $R$. We begin by proving the next Lemma.

Lemma 3.4. The following problem

$$
\left\{\begin{array}{l}
v_{t}(t, x)-v_{x x}(t, x)+(g(x) v(t, x))_{x}+(d(x)+\mu) v(t, x)=0, \quad t \geq 0, \quad x \in(0, R), \\
g(0) v(t, 0)-v_{x}(t, 0)=\alpha \int_{0}^{R} B(y) v(t, y) d y+\gamma e^{-\mu t}, \text { and } v(t, R)=0, \\
v(0, x)=n_{0}(x) .
\end{array}\right.
$$

has a unique positive solution $v_{R}$, moreover if $R_{1} \leq R_{2}$, then $v_{R_{1}} \leq v_{R_{2}}$. 
Proof. To prove the existence of a positive solution to (3.5) we use the Piccard Banach fixed point theorem in the Banach space $X_{R}=\mathcal{C}\left([0, T], L^{1}(0, R)\right)$ with $\|\phi\|_{X_{R}}=\sup _{t \in[0, T]} \int_{0}^{R}|\phi(t, x)| d x$ and $\mu>0$ to be chosen later. More precisely given $m \in X_{R}$, we define $v:=S(m)$ as the solution to problem

$$
\left\{\begin{array}{l}
v_{t}(t, x)-v_{x x}(t, x)+(g(x) v(t, x))_{x}+(d(x)+\mu) v(t, x)=0, \text { in }(0, T) \times(0, R), \\
g(0) v(t, 0)-v_{x}(t, 0)=\alpha \int_{0}^{R} B(x) m(t, x) d x+\gamma e^{-\mu t}, \quad v(t, R)=0, \\
v(0, x)=n_{0}(x), \quad x \in(0, R) .
\end{array}\right.
$$

It is clear that $\left.v \in L^{2}\left(0, T ; W^{1,2}(0, R)\right)\right) \cap X_{R}$. For $m_{1}, m_{2} \in X_{R}$ we consider $v_{1}:=S\left(m_{1}\right)$, $v_{2}:=S\left(m_{2}\right)$. Let $v=v_{1}-v_{2}, m=m_{1}-m_{2}$, it follows that

$$
\left\{\begin{array}{l}
v_{t}(t, x)-v_{x x}(t, x)+(g(x) v(t, x))_{x}+(d(x)+\mu) v(t, x)=0, \quad \text { in }(0, T) \times(0, R), \\
g(0) v(t, 0)-v_{x}(t, 0)=\alpha \int_{0}^{R} B(x) m(t, x) d x, \quad v(t, R)=0, \\
v(0, x)=0 .
\end{array}\right.
$$

Multiplying equation (3.7) by $\operatorname{sgn}(v)$ and integrating in $x$,

$$
\frac{d}{d t} \int_{0}^{R}|v(t, x)| d x+\int_{0}^{R}(d(x)+\mu)|v(t, x)| d x \leq \alpha \int_{0}^{R} B(y)|m(t, y)| d y,
$$

after integration over $\left(0, T_{1}\right), T_{1} \leq T$,

$$
\int_{0}^{R}\left|v\left(T_{1}, x\right)\right| d x+\int_{0}^{T_{1}} \int_{0}^{R}(d(x)+\mu)|v(t, x)| d x \leq \alpha B_{M} T\|m\|_{X_{R}}
$$

and then $\|v\|_{X_{R}} \leq \alpha B_{M} T\|m\|_{X_{R}}$. Hence choosing $T$ such $\alpha T B_{M}<1$ we obtain that the operator $S$ is strict contraction in Banach space $X_{R}$ which proves the existence of a unique fixed point $v_{R}$. As usual we can iterate the operator on $[T, 2 T],[2 T, 3 T], \ldots$ since the condition on $T$ does not depend on the iteration. With this iteration process, we have built a solution in $\mathcal{C}\left([0, T], L^{1}(0, R)\right)$. The positivity of the solution, is a simple consequence of the above comparison lemma. The strong maximum principle allows us to get the strict positivity of $v_{R}$. Let $R_{1} \leq R_{2}$, and consider the corresponding solutions $v_{R_{1}}$ and $v_{R_{2}}$. It is clear that $v_{R_{2}}$ is a supersolution to the $v_{R_{1}}$-problem. Hence using the comparison principle we obtain that $v_{R_{1}} \leq v_{R_{2}}$. Therefore the result follows.

We return now to prove the first existence lemma

Proof of Lemma 3.3. Let $v_{R}$ the solution to problem (3.5) obtained above. We define $v_{R}$ for $(t, x) \in(0, T) \times(R, \infty)$ by setting set $v_{R}(t, x)=0$. Then $v_{R} \in X \equiv L^{1}\left((0, T) \times \mathbb{R}^{+}\right) \cap$ $L^{2}\left(0, T ; W^{1,2}\left(\mathbb{R}^{+}\right)\right)$. We know that

$$
\frac{d}{d t} \int_{0}^{R} v_{R}(t, x) d x+\int_{0}^{R}(d(x)+\mu) v_{R}(t, x) d x \leq \alpha B_{M} \int_{0}^{R} v_{R}(t, y) d y+\gamma e^{-\mu t} .
$$

Choosing $\mu>\alpha B_{M}$, it follows that

$$
\frac{d}{d t} \int_{0}^{R} v_{R}(t, x) d x+\int_{0}^{R}\left(d(x)+\mu-B_{M}\right) v_{R}(t, x) d x \leq \gamma e^{-\mu t} .
$$


Hence from Gronwall lemma we get

$$
\int_{0}^{R} v_{R}(t, x) d x \leq C
$$

with $C>0$. Thus we conclude that $v_{R}$ is uniformly bounded in $L^{1}\left((0, T) \times \mathbb{R}^{+}\right)$.

Therefore using the monotonicity of the sequence $\left\{v_{R}\right\}$ we get the existence of $v \in L^{1}\left((0, T) \times \mathbb{R}^{+}\right)$ such that $v_{R} \uparrow v$ as $R \rightarrow \infty$.

Taking $v_{R}$ as a test function in (3.5), after integration, there result that

$$
\frac{1}{2} \frac{d}{d t} \int_{0}^{R} v_{R}^{2}(t, x) d x+\int_{0}^{R}\left(d(x)+\frac{1}{2} g^{\prime}(x)+\mu\right)\left|v_{R}(t, x)\right|^{2} d x+\int_{0}^{R}\left|\left(v_{R}\right)_{x}(t, x)\right|^{2} d x \leq C,
$$

by integration in the time we have

$\int_{0}^{R}\left|v_{R}(t, x)\right|^{2} d x+\int_{0}^{t} \int_{0}^{R}\left(d(x)+\frac{1}{2} g^{\prime}(x)+\mu\right)\left|v_{R}(t, x)\right|^{2} d x d t+\int_{0}^{t} \int_{0}^{R}\left|\left(v_{R}\right)_{x}(t, x)\right|^{2} d x \leq \frac{1}{2}\left\|n_{0}\right\|_{L^{2}}^{2}+C t$,

where $C$ is a positive constant. Thus for $\mu$ so large, we have $\left\|v_{R}\right\|_{L^{2}\left(0, T ; W^{1,2}\left(\mathbb{R}^{+}\right)\right)} \leq C$ and then $v_{R} \rightarrow v$ weakly in $L^{2}\left(0, T ; W^{1,2}\left(\mathbb{R}^{+}\right)\right)$.

Therefore classical regularity result of parabolic equation allows us to pass to the limit in the boundary condition to conclude that $v$ solves problem (3.1). To get the uniqueness result for problem (3.1), we suppose that $v_{1}, v_{2} \in X$ are two solution of (3.1), then $w=v_{1}-v_{2}$, solves (1.1) with $w(0, x)=0$. Hence multiply the equation of $w$ by $\operatorname{sgn}(w)$, after integrating over $(0, \infty)$, we obtain

$$
\int_{0}^{\infty}|w|_{t}(t, x) d x+\int_{0}^{\infty}(d(x)+\mu)|w(t, x)| d x \leq \alpha B_{M} \int_{0}^{\infty}|w(t, x)| d x
$$

thus

$$
\int_{0}^{\infty}|w|_{t}(t, x) d x+C_{1} \int_{0}^{\infty}|w(t, x)| d x \leq 0
$$

and then by the Gronwall lemma we conclude that $w=0$. Hence the result follows.

proof of Theorem 3.1. We consider the following approximated problem

$$
\left\{\begin{array}{l}
n_{t}^{k}(t, x)-n_{x x}^{k}(t, x)+\left(g(x) n^{k}(t, x)\right)_{x}+d(x) n^{k}(t, x)=0, \quad t \geq 0, \quad x \geq 0 \\
g(0) n^{k}(t, 0)-n_{x}^{k}(t, 0)=f\left(\int_{0}^{\infty} B(x) n^{k-1}(t, x) d x\right), \quad \text { and } n^{k}(0, x)=n_{0}(x) \\
n^{0}(t, x)=v(t, x),
\end{array}\right.
$$

with $v$ is solution to problem (3.1). We easily proved that $0 \leq n^{k} \leq v$ ( since 0 is subsolution to (1.2)).

Now multiplying the solution of $(3.9)$ by $n^{k}$ and integrating over $(0, \infty)$, we have

$$
\begin{aligned}
\frac{1}{2} \frac{d}{d t} \int_{0}^{\infty}\left|n^{k}\right|^{2} d x+\int_{0}^{\infty} d(x)\left|n^{k}(t, x)\right|^{2} d x+\int_{0}^{\infty}\left|\left(n^{k}\right)_{x}(t, x)\right|^{2} d x & \leq \frac{1}{2} f^{2}\left(\int_{0}^{\infty} B(x) n^{k-1}(t, x) d x\right) \\
& \leq \alpha^{2}\left(\int_{0}^{\infty} B(x) v(t, x) d x\right)^{2}+\gamma^{2}+\frac{1}{2} \int_{0}^{\infty}\left|g^{\prime}(x)\right| v^{2}(t, x) d x
\end{aligned}
$$


then

$$
n^{k} \rightarrow n \text { in } L^{2}\left((0, T) ; W^{1,2}\left(\mathbb{R}^{+}\right)\right)
$$

hence $n$ is solution to problem (1.2). The uniqueness is proved easily by the above comparison principle.

proof of Theorem 2.2. Consider $n_{0} \in L^{1}\left(\mathbb{R}^{+}, e^{-G(x)} d x\right)$. By density we can find a nondecreasing sequence $n_{0}^{k} \in L^{1}\left(\mathbb{R}^{+}\right) \bigcap L^{2}\left(\mathbb{R}^{+}\right)$such that $n_{0}^{k} \rightarrow n_{0}$ in $L^{1}\left(\mathbb{R}^{+}, e^{-G(x)} d x\right)$. We denote by $n^{(k)}(t, x)$ the corresponding solution to problem (1.2). We compute for the solution $w=\left(n^{(k)}-n^{(p)}\right) e^{-\mu t}$, it follows

$$
\left\{\begin{array}{l}
e^{-G(x)} w_{t}(t, x)-\left(e^{-G(x)} w_{x}(t, x)\right)_{x}+d(x) e^{-G(x)} w(t, x)=0 \quad t \geq 0, \quad x \geq 0 \\
g(0) w(t, 0)-w_{x}(t, 0)=\left(f\left(\int_{0}^{\infty} B(x) n^{(k)}(t, x) d x\right)-f\left(\int_{0}^{\infty} B(x) n^{(p)}(t, x) d x\right)\right) e^{-\mu t}, \\
w(0, x)=n_{0}^{(k)}(x)-n_{0}^{(p)}(x),
\end{array}\right.
$$

setting the truncated function

$$
T_{1}(w)=\left\{\begin{array}{l}
w \text { if }|w| \leq 1 \\
\frac{w}{|w|} \text { if }|w|>1
\end{array}\right.
$$

and $\Theta(s)=\int_{0}^{s} T_{1}(\sigma) d \sigma$. Multiplying the equation of problem (3.10) by $T_{1}(w)$ and integrating,

$$
\begin{aligned}
& \frac{d}{d t} \int_{0}^{\infty} e^{-G(x)} \Theta(w(t, x)) d x+\int_{0}^{\infty} e^{-G(x)} w_{x}(t, x)\left(T_{1}(w(t, x))\right)_{x} d x \\
+ & \int_{0}^{\infty} e^{-G(x)}\left(d(x)+g^{\prime}(x)+\mu\right) w(t, x) T_{1}(w(t, x)) d x=-w_{x}(t, 0) T_{1}(w(t, 0))
\end{aligned}
$$

thus

$$
\frac{d}{d t} \int_{0}^{\infty} e^{-G(x)} \Theta(w(t, x)) d x \leq\left(f^{\prime}(\theta(t)) \int_{0}^{\infty} B(x) w(t, x) d x-w(t, 0)\right) T_{1}(w(t, 0)),
$$

with $\theta(t)$ is a value between $\int_{0}^{\infty} B(x) n^{(k)}(t, x) d x$ and $\int_{0}^{\infty} B(x) n^{(p)}(t, x) d x$, which have uniformly bounded. Now, we have by integrating over $(0, t)$,

$$
\begin{aligned}
\int_{0}^{\infty} e^{-G(x)} \Theta(w(t, x)) d x & \leq C \int_{0}^{t} \int_{0}^{\infty}|w(s, x)| d x d s+\int_{0}^{\infty} e^{-G(x)} \Theta(w(0, x)) d x, \\
& \leq C \int_{0}^{t} \int_{0}^{\infty}|w(s, x)| d x d s \\
& +\int_{\left\{x \in \mathbb{R}^{+},|w(0, x)|>1\right\}} e^{-G(x)}|w(0, x)| d x+\frac{1}{2} \int_{\left\{x \in \mathbb{R}^{+},|w(0, x)| \leq 1\right\}} e^{-G(x)|w(0, x)|^{2} d x .}
\end{aligned}
$$

By integrating the equation (1.2) and again Gronwall lemma, we obtain

$$
\int_{0}^{\infty} \int_{0}^{\infty} n^{(k)}(t, x) d x \leq C .
$$

Therefore using the monotonicity of the sequence $n^{(k)}$ we get existence of $n \in L^{1}\left(\mathbb{R}^{+} \times \mathbb{R}^{+}\right)$such that $n^{(k)} \longrightarrow n$ as $k \longrightarrow \infty$ in $L^{1}\left(\mathbb{R}^{+} \times \mathbb{R}^{+}\right)$. Henceforth $w$ is a Cauchy sequence in $L^{1}\left((0, T) \times \mathbb{R}^{+}\right)$. In other hand,

$$
\int_{0}^{\infty} e^{-G(x)} \Theta(w(t, x)) d x=\int_{\left\{x \in \mathbb{R}^{+},|w|>1\right\}} e^{-G(x)}|w| d x+\frac{1}{2} \int_{\left\{x \in \mathbb{R}^{+},|w| \leq 1\right\}} e^{-G(x)}|w|^{2} d x,
$$


and, by Holder inequality, we find

$$
\int_{\left\{x \in \mathbb{R}^{+},|w| \leq 1\right\}} e^{-G(x)}|w| d x \leq\left(\int_{\left\{x \in \mathbb{R}^{+},|w| \leq 1\right\}} e^{-G(x)}|w|^{2} d x\right)^{\frac{1}{2}}\left(\int_{0}^{\infty} e^{-G(x)} d x\right)^{\frac{1}{2}} .
$$

Combining these above results and by the hypothesis of the initial data, we prove that $n^{(k)}$ is a Cauchy sequence $C\left([0, T], L^{1}\left(\mathbb{R}^{+}, e^{-G(x)} d x\right)\right)$. Therefore it's converges to function $n \in C\left([0, T], L^{1}\left(\mathbb{R}^{+}, e^{-G(x)} d x\right)\right)$. The result is proved.

\section{Convergence to a stationary problem}

In this section we prove that under some hypotheses on the growth of $f$ and the initial data, we have the convergence towards a stationary solution.

Noticing that the trivial solution is not stable (see section 1) for all positive (non trivial) initial datum $n_{0} \in L^{1}\left(\mathbb{R}^{+}, e^{-G(x)} d x\right), n(t, x)$ solution to (1.2) is also solution to the problem

$$
\left\{\begin{array}{l}
n_{t}(t, x)-n_{x x}(t, x)+(g(x) n(t, x))_{x}+d(x) n(t, x)=0 \quad t \geq 0, \quad x \geq 0 \\
g(0) n(t, 0)-n_{x}(t, 0)=\tilde{f}\left(\int_{0}^{\infty} B(x) n(t, x) d x\right), \text { and } n(0, x)=n_{0}(x) \in L_{+}^{1}\left(\mathbb{R}^{+}\right),
\end{array}\right.
$$

where $\tilde{f}(x)=f\left(\max \left(x, \min \left(s_{0}, \int_{0}^{\infty} n_{0}(y) d y\right)\right)\right.$ is strictly positive and $\tilde{f}(0) \neq 0$.

We consider the following problem

$$
\left\{\begin{array}{l}
-u^{\prime \prime}(x)-g(x) u^{\prime}(x)+d(x) u(x)=B(x), \quad x \geq 0 \\
u^{\prime}(0)=0 \\
u \in W^{1, \infty}\left(\mathbb{R}^{+}\right)
\end{array}\right.
$$

We begin by stating the existence, positivity and boundedness of the solution to problem (4.2).

Lemma 4.1. Under the hypotheses (2.1),(2.5), the problem (4.2) has a unique positive solution $u$. In addition we have

$$
0<u(x) \leq \frac{B_{M}}{d_{m}}, \quad \forall x \geq 0 .
$$

Proof. In order to prove the existence of solution, we argue by approximation, namely we consider the case of bounded domain $[0, R]$ and then we pass to the limit in $R$. So let us define the following problem

$$
\left\{\begin{array}{l}
-u_{R}^{\prime \prime}(x)-g(x) u_{R}^{\prime}(x)+d(x) u_{R}(x)=B(x), \quad x \in(0, R), \\
u_{R}^{\prime}(0)=0 \\
u_{R}(R)=0
\end{array}\right.
$$

Using (for instance) Lax-Milgram method and multiplying by the negative part of $u_{R}$, the problem (4.4) has a unique positive solution. Moreover by principle maximum the solution $u_{R}$ is strictly positive in $[0, R)$. Now remarking that $\bar{u}_{R}=\frac{B_{M}}{d_{m}}$ is supersolution of problem (4.4). Consequently by classical comparison principle we prove the inequalities $0<u_{R}(x) \leq \frac{B_{M}}{d_{m}}$. In addition, notice that (again by principle comparison) the sequence $u_{R}$ is nondecreasing with respect to $R$, therefore $u_{R}$ (see as an extension by 0 outside $(0, R)$ ) converge to strictly positive function $u$ solution of problem (4.2). Now we suppose that $v$ is another solution of problem (4.2) then $v$ is a supersolution of problem $(4.4)$ in $(0, R)(v(R)>0)$, thus $u_{R} \leq v$ and by passing to a limit, we can prove that $u$ is the minimal solution of problem (4.2). 
Moreover, rewriting the equation (4.2) as

$$
-\left(u^{\prime} e^{G(x)}\right)^{\prime}+d(x) e^{G(x)} u(x)=B(x) e^{G(x)},
$$

by integration, we obtain

$$
u^{\prime}(x)=e^{-G(x)} \int_{0}^{x} \frac{d(s)}{g(s)} g(s) e^{G(s)} u(s) d s-e^{-G(x)} \int_{0}^{x} \frac{B(s)}{g(s)} g(s) e^{G(s)} d s,
$$

thus

$$
\left|u^{\prime}(x)\right| \leq\left(\frac{d_{M}}{g_{m}}+\frac{B_{M}}{g_{m}}\right)\left(1-e^{-G(x)}\right) .
$$

The existence of the minimal solution in $W^{1, \infty}\left(\mathbb{R}^{+}\right)$is proved. Concerning the uniqueness, we set $w=v-u \geq 0$, thus $w$ satisfy the following problem

$$
\left\{\begin{array}{l}
-\left(w^{\prime} e^{G(x)}\right)^{\prime}+d(x) e^{G(x)} w(x)=0, \quad x \geq 0, \\
w^{\prime}(0)=0, \\
w \in W^{1, \infty}\left(\mathbb{R}^{+}\right) .
\end{array}\right.
$$

Multiplying the equation of problem (4.6) by a test function $\phi(x)=e^{-\Gamma G(x)}$, with $\Gamma>1$ is a constant to be chosen later, and integrating by part, we obtain

$$
-w(0) \phi^{\prime}(0)+\int_{0}^{\infty}\left(d(x)+\Gamma g^{\prime}(x)-\Gamma(\Gamma-1) g^{2}(x)\right) \phi(x) e^{G(x)} w(x) d x=0 .
$$

Now choosing $\Gamma$ such that

$$
d(x)+\Gamma g^{\prime}(x)-\Gamma(\Gamma-1) g^{2}(x) \geq \delta>0,
$$

and since $\phi^{\prime}(0)<0$ then $w(x)=0$. Hence the lemma is proved

In order to analyze the asymptotic behavior of the solution to problem (1.2), we need to show the existence, uniqueness and positivity of the stationary solution to problem (2.8). Indeed we have the following lemma

Lemma 4.2. The problem (2.8) has a positive solution $N$ with $N \in W^{2,2}\left(\mathbb{R}^{+}\right)$if and only if there exists a positive constant $\Gamma$ such that

$$
f(\Gamma)=\frac{1}{u(0)} \Gamma
$$

with $u$ is solution to problem (4.2). Moreover $\Gamma=\int_{0}^{\infty} B(x) N(x) d x$.

Proof. Multiplying the equation of problem (2.8) by $u$ solution to the problem (4.2) and integrating over $(0, \infty)$ we have

$$
\int_{0}^{\infty} B(x) N(x) d x=f\left(\int_{0}^{\infty} B(x) N(x) d x\right) u(0),
$$

Now, let $N$ be the solution of the following problem

$$
\left\{\begin{array}{l}
-N^{\prime \prime}(x)+(g N)^{\prime}(x)+d(x) N(x)=0, \quad x \geq 0, \\
g(0) N(0)-N^{\prime}(0)=f(\Gamma), \\
\int_{0}^{\infty} N(x) d x<\infty, \text { and } N \geq 0 .
\end{array}\right.
$$


Multiplying by $u$ and integrating, we obtain

$$
\int_{0}^{\infty} B(x) N(x) d x=f(\Gamma) u(0)
$$

thus by hypothesis (4.7), we have $\Gamma=\int_{0}^{\infty} B(x) N(x) d x$.

In order to prove the asymptotic behavior of the solution to problem (1.2), we consider the following auxiliary problems, setting $\tilde{u}(x):=V(x)+C N(x)$,

$$
\left\{\begin{array}{l}
U_{t}(t, x)-U_{x x}(t, x)+(g(x) U(t, x))_{x}+d(x) U(t, x)=0, \quad t \geq 0, \quad x \geq 0 \\
g(0) U(t, 0)-U_{x}(t, 0)=\tilde{f}\left(\int_{0}^{\infty} B(x) U(t, x) d x\right), \quad \text { and } U(0, x)=\tilde{u}
\end{array}\right.
$$

with $C \geq 1$ to be chosen later. $N$ is the solution of the stationary problem (2.8) and $V$ satisfies

$$
\left\{\begin{array}{l}
-V^{\prime \prime}(x)+(g V)^{\prime}(x)+d(x) V(x)=0, \quad x \geq 0 \\
g(0) V(0)-V^{\prime}(0)=\alpha \int_{0}^{\infty} B(x) V(x) d x+\gamma \text { and } \int_{0}^{\infty} V(x) d x<\infty .
\end{array}\right.
$$

First of all, we begin by studying the above stationary problem (4.12). Indeed we have the following Lemma which the proof is the same as Lemma 4.2. However we give a somehow different proof.

Lemma 4.3. The problem (4.12) has a unique positive solution if and only if $\alpha<\frac{1}{u(0)}$, and $\gamma>0$

Proof. By multiplying the equation of problem (4.12) by $u$ the minimal solution of problem (4.2) and integrating, we obtain

$$
(1-\alpha u(0)) \int_{0}^{\infty} B(x) V(x) d x=\gamma u(0),
$$

on the other hand, we define the operator $A$ from $L^{1}\left(\mathbb{R}^{+}, B(x) d x\right)$ to $L^{1}\left(\mathbb{R}^{+}, B(x) d x\right)$ such that for each function $m$ we set $A(m)=V$, with $V$ satisfies

$$
\left\{\begin{array}{l}
-V^{\prime \prime}(x)+(g V)^{\prime}(x)+d(x) V(x)=0, \quad x \geq 0, \\
g(0) V(0)-V^{\prime}(0)=\frac{1}{u(0)} \int_{0}^{\infty} B(x) m(x) d x+\gamma,
\end{array}\right.
$$

thus by applying the Banach Piccard fixed point theorem we can easily prove the existence, uniqueness of the solution to problem (4.12).

Lemma 4.4. Assume that (2.3), (2.4) and $n_{0}(x) \leq C N(x)$. Let $\bar{U}(t, x)$ and $\underline{U}(t, x)$ be the solutions of the problem (4.11), with the initial condition is respectively $\tilde{u}$ and 0 . Suppose that $\alpha<\frac{1}{u(0)}$ and $\gamma>0$. Then we have the following inequalities

$$
0 \leq \underline{U}(t, x) \leq n(t, x) \leq \bar{U}(t, x) \leq \tilde{u}(x), \forall t \geq 0,
$$

Furthermore $\bar{U}(t, x)$ resp $(\underline{U}(t, x))$ is nonincreasing in $t$ (is nondecreasing in $t$ ).

Proof. First of all, remarking that the assumptions (2.3) and (2.4) give the existence of $\Gamma>0$ solution to $f(\Gamma)=\frac{1}{u(0)} \Gamma$. Setting $w(t, x)=\bar{U}(t, x)-\tilde{u}(x)$, then $w$ satisfies 


$$
\left\{\begin{array}{l}
w_{t}(t, x)-w_{x x}(t, x)+(g(x) w(t, x))_{x}+d(x) w(t, x)=0, \quad t \geq 0, \quad x \geq 0 \\
g(0) w(t, 0)-w_{x}(t, 0) \leq \alpha \int_{0}^{\infty} B(x) w(t, x) d x \\
w(0, x)=0
\end{array}\right.
$$

As a simple consequence of comparison principle, we get the desired result. Similarly by setting $w(t, x)=n(t, x)-\bar{U}(t, x)$ we have

$$
\left\{\begin{array}{l}
w_{t}(t, x)-w_{x x}(t, x)+(g(x) w(t, x))_{x}+d(x) w(t, x)=0, \quad t \geq 0, \quad x \geq 0 \\
g(0) w(t, 0)-w_{x}(t, 0)=\tilde{f}\left(\int_{0}^{\infty} B(x) n(t, x) d x\right)-\tilde{f}\left(\int_{0}^{\infty} B(x) \bar{U}(t, x) d x\right)=\tilde{f}^{\prime}(\theta(t)) \int_{0}^{\infty} B(x) w(t, x) d x, \\
w(0, x) \leq 0
\end{array}\right.
$$

where $\theta(t)$ is a value between $\int_{0}^{\infty} B(x) n(t, x) d x$ and $\int_{0}^{\infty} B(x) \bar{U}(t, x) d x$. Remarking that $\theta(t)$ is uniformly bounded $(\bar{U} \leq \tilde{u})$ and so using the fact that $f$ is nondecreasing, we have

$$
\left\{\begin{array}{l}
w_{t}(t, x)-w_{x x}(t, x)+(g(x) w(t, x))_{x}+d(x) w(t, x)=0, \quad t \geq 0, \quad x \geq 0 \\
g(0) w(t, 0)-w_{x}(t, 0) \leq c \int_{0}^{\infty} B(x) w^{+}(t, x) d x \\
w(0, x) \leq 0
\end{array}\right.
$$

where the positive constant $c$ satisfying $f^{\prime}(\theta(t)) \leq c$. Again by comparison principle we obtain $w \leq 0$. Concerning the monotonicity of $\bar{U}$, by setting $w=\bar{U}\left(t+t_{1}, x\right)-\bar{U}(t, x)$ for all $t_{1}$ positive, so $w$ satisfies the problem (4.16) with $w(0, x)=U\left(t_{1}, x\right)-\tilde{u}(x) \leq 0$, and so another time by comparison principle, we have the monotonicity of $\bar{U}(t, x)$.

Now we are able to prove the theorem 2.3. Indeed,

\section{Proof of theorem 2.3.}

First we know that $\bar{U}(t, x)$ converges to a limit, so setting $\bar{U}(t, x) \rightarrow \bar{U}_{s}(x)$ as $t \rightarrow \infty$. Consider the boundary- value problem

$$
\left\{\begin{array}{l}
-v^{\prime \prime}(x)+(g v)^{\prime}(x)+d(x) v(x)=0, \quad x \geq 0 \\
g(0) v(0)-v^{\prime}(0)=\tilde{f}\left(\int_{0}^{\infty} B(y) \bar{U}_{s}(y) d y\right)
\end{array}\right.
$$

Now by setting $w(t, x)=\bar{U}(t, x)-v(x)$, and $W(x)=\tilde{u}(x)-v(x)$. We claim that $W \geq 0$. Indeed $W$ satisfies

$$
\left\{\begin{array}{l}
-W^{\prime \prime}(x)+(g W)^{\prime}(x)+d(x) W(x) \geq 0, \quad x \geq 0 \\
g(0) W(0)-W^{\prime}(0) \geq 0
\end{array}\right.
$$

then by the simple comparison principle we conclude the claim. Using the same idea as above and the fact that $f$ is nondecreasing we can prove that $w(t, x) \geq 0$.

Multiplying the solution of problem (4.16) by $w$ and integrating over $(t, t+1) \times(0, \infty)$,

$$
\begin{aligned}
& \int_{t}^{t+1} \int_{0}^{\infty} \frac{\partial}{\partial s} w(s, x) w(s, x) d x+\int_{t}^{t+1} \int_{0}^{\infty}\left(d(x)+\frac{1}{2} g^{\prime}(x)\right)|w(s, x)|^{2} d x d s+\int_{t}^{t+1} \int_{0}^{\infty}\left|w_{x}(s, x)\right|^{2} d x d s \\
& \leq \frac{1}{2} \int_{t}^{t+1}\left(\tilde{f}\left(\int_{0}^{\infty} B(x) \bar{U}(s, x) d x\right)-\tilde{f}\left(\int_{0}^{\infty} B(x) \bar{U}_{s}(x) d x\right)\right)^{2} d s .
\end{aligned}
$$


Thus using the fact that $w(t,$.$) is nonincreasing in t$, we obtain

$$
\begin{aligned}
\frac{1}{2} \int_{0}^{\infty}\left(|w(t+1, x)|^{2}\right. & \left.-|w(t, x)|^{2}\right) d x+\int_{0}^{\infty}\left(d(x)+\frac{1}{2} g^{\prime}(x)\right)|w(t+1, x)|^{2} d x \\
& \leq \frac{1}{2}\left(\tilde{f}\left(\int_{0}^{\infty} B(x) \bar{U}(t, x) d x\right)-\tilde{f}\left(\int_{0}^{\infty} B(x) \bar{U}_{s}(x) d x\right)\right)^{2} .
\end{aligned}
$$

In addition, $\bar{U}(t, x) \rightarrow \bar{U}_{s}(x)$ as $t \rightarrow \infty$ in $L^{1}\left(\mathbb{R}^{+}\right)$and the continuity of $\tilde{f}$ we have

$$
\tilde{f}\left(\int_{0}^{\infty} B(x) \bar{U}(t, x) d x\right) \rightarrow \tilde{f}\left(\int_{0}^{\infty} B(x) \bar{U}_{s}(x) d x\right)
$$

as $t \rightarrow \infty$.

By passing to the limit as $t \longrightarrow \infty$ and combining these above results we obtain

$w(t,.) \rightarrow 0$ in $L^{2}\left(\mathbb{R}^{+}\right)$and so $v=\bar{U}_{s}$, and consequently $\bar{U}_{s}=N$. In order to prove that $\bar{U}_{s}$ is the maximal solution of stationary problem (2.8), we set $w=\bar{U}(t, x)-N(x)$, with $N$ is any solution of problem (2.8). Applying the Lemma 3.2, we can prove that $\bar{U}(t, x) \geq N(x)$. Now by passing to the limit, we obtain the result. Applying the same idea as above, we can prove that $\underline{U}(t, x) \rightarrow \underline{N}(x)$ as $t \rightarrow \infty$. hence we conclude the proof of the theorem.

We have the following corollary concerning the case, where the problem (2.8) has a multiple non trivial stationary solution.

Corollary 4.5. Suppose that the problem (2.8) admits $n$ nontrivial steady states called $N_{i}(x)$ for $1 \leq i \leq n$. Assume that the initial condition satisfy either $N_{i}(x) \supsetneqq n_{0}(x) \supsetneqq N_{i+1}(x)$ for $1 \leq i \leq n-1$ or $N_{n}(x) \leq n_{0}(x) \leq C N_{n}(x)$, resp $\left(n_{0}(x) \leq N_{1}(x)\right)$ and $n_{0}$ is a supersolution, resp (subsolution) of problem (2.8). Then the solution of problem (1.2) converge to $N_{i}(x) \operatorname{resp}\left(N_{i+1}(x)\right)$.

Proof. Since $n_{0}$ is supersolution of problem (2.8), then by principle comparison, we have $n(t, x) \leq$ $n_{0}(x)$ and $n(t, x)$ is nonincreasing with respect to $t$. Consequently its admits a limit. By following the proof of theorem 2.3 , we get the result.

\section{BLOW UP / ExTINCTION}

In this section we are concerned with a blow up (resp. extinction) of the solution to problem (1.2) under some assumptions on the growth of $f$.

Theorem 5.1. Under the existence of $M_{1}$ and $M_{2}$ such that

$$
\alpha x+\gamma \leq f(x) \leq M_{1} x+M_{2},
$$

with $\alpha \geq \frac{1}{u(0)}$ and $\gamma>0$ or $\alpha>\frac{1}{u(0)}$ and $\gamma=0$, the solution $n$ to (1.2) satisfies

$$
\lim _{t \rightarrow \infty} \int_{0}^{\infty} n(t, x) d x=+\infty
$$

Proof. Multiplying the equation (1.2) by $u$ and integrating, we have 


$$
\begin{aligned}
\int_{0}^{\infty} n_{t}(t, x) u(x) d x & =\int_{0}^{\infty}\left(n_{x x}-(g n)_{x}-d(x) n\right) u(x) d x \\
& =u(0)\left(n(t, 0)-n_{x}(t, 0)\right)+\int_{0}^{\infty} n(t, x)\left(u^{\prime \prime}(x)+g(x) u^{\prime}(x)-d(x) u(x)\right) d x, \\
& =u(0) f\left(\int_{0}^{\infty} B(x) n(t, x) d x\right)-\int_{0}^{\infty} B(x) n(t, x) d x, \\
& \left.\geq u(0)\left(\left(\alpha-\frac{1}{u(0)}\right) \int_{0}^{\infty} B(x) n(t, x) d x\right)+\gamma\right),
\end{aligned}
$$

Hence, using Lemma 4.1 we get the result.

We have directly the following extinction result.

Theorem 5.2. Under the existence of $M_{1}$ such that

$$
f(x) \leq M_{1} x,
$$

with $M_{1}<\frac{1}{u(0)}$ the solution $n$ to (1.2) satisfies

$$
\lim _{t \rightarrow \infty} \int_{0}^{\infty} n(t, x) d x=0 .
$$

\section{Numerical Simulation}

We present some examples to illustrate the result of the previous sections, namely the convergence toward the positive steady states. For almost all these examples we suppose that the recruitment term $f(x)=\sqrt{x}$, and the transport term $g(x)=1$ which satisfy the assumptions of the theorem 2.3. The initial conditions is assumed to be equal to 1 . We compute numerical solution to the system (1.2) using finite difference with Dirichlet condition at the right boundary. The birth and death terms are given by $B(x)=5 e^{-x}+10\left(1-e^{-x}\right)$, and $d(x)=2 e^{-x}+4\left(1-e^{-x}\right)$. 


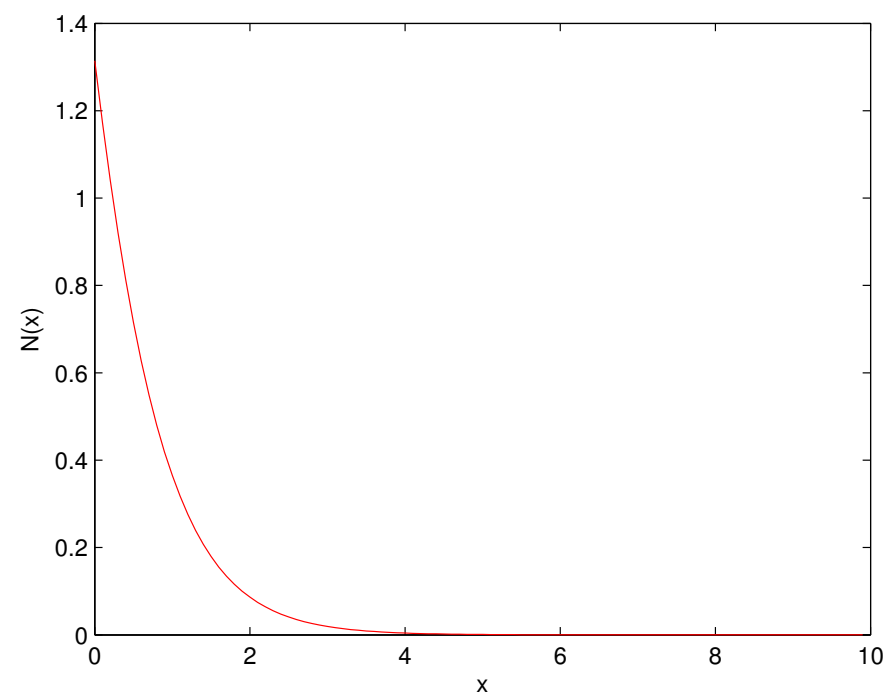

FiguRE 1. : The numerical simulation of the steady state solution to problem (2.8).

The next figure illustrate the Theorem 2.3, namely the convergence of the solution to problem (1.2) towards the nontrivial solution of problem (2.8). 


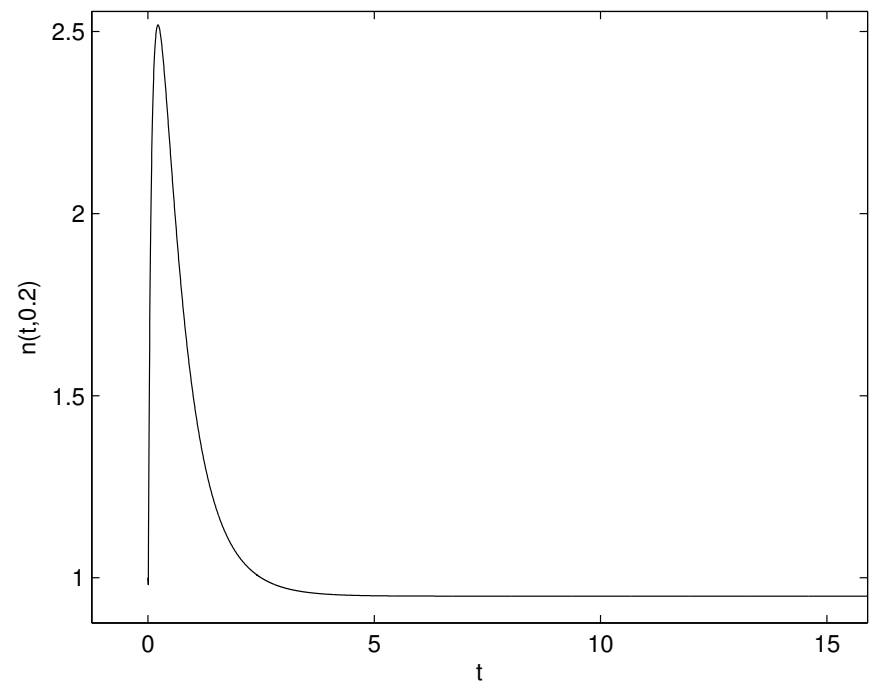

Figure 2. : $x=0.2$. The numerical simulation of the solution to problem (1.2). Here we fix an age $x=0.2$, we compute $n(t, x)$ and we observe the convergence in long time asymptotic.

\section{Conclusion}

We have proved the existence, uniqueness and study the dynamics of solutions to the nonlinear partial differential equation (1.2).

We notice that the assumptions (2.3)-(2.4) on the growth of the nonlinear birth rate function $f$ depends on the supremum and infimum of the birth rate $B$ and death rate $d$. In the general case, the birth rate vanishes when the age is too small (immature) or too large (do not give birth when the individual is too old). In the same way, the biological age of individuals is "limited" and so the death rate can goes to infinity when the age is too large. In figure 4, we conjecture that we can obtain the same result, even if the birth term vanishes and the death term goes to infinity, but not too fast, (behaves as $x$ in infinity for example). Therefore, it will be interesting to find assumptions on $f$ which extend the convergence result when the birth rate and death rate are nonnegative and not necessarily bounded.

\section{REFERENCES}

[1] B. Abdellaoui, T.M.Touaoula, Decay solution for the renewal equation with diffusion. Nonlinear Differ. Equ. Appl. (Nodea) vol. 17, 2010, 271-288.

[2] B. Basse, B. C. Baguley, E.S. Marshall, W. R. Joseph, B. van Brunt, G. Wake, D. J. N. Wall, A mathematical model for analysis of the cell cycle in cell lines derived from human tumors. J. Math. Biol. Vol. 47 (4), 2003, 295-312.

[3] J. W. Brewer, The age-dependent eigenfunctions of certain Kolmogorov equations of engineering, economics, and biology Appl. Math. Modelling, Vol. 13, 1989, 47-57. 


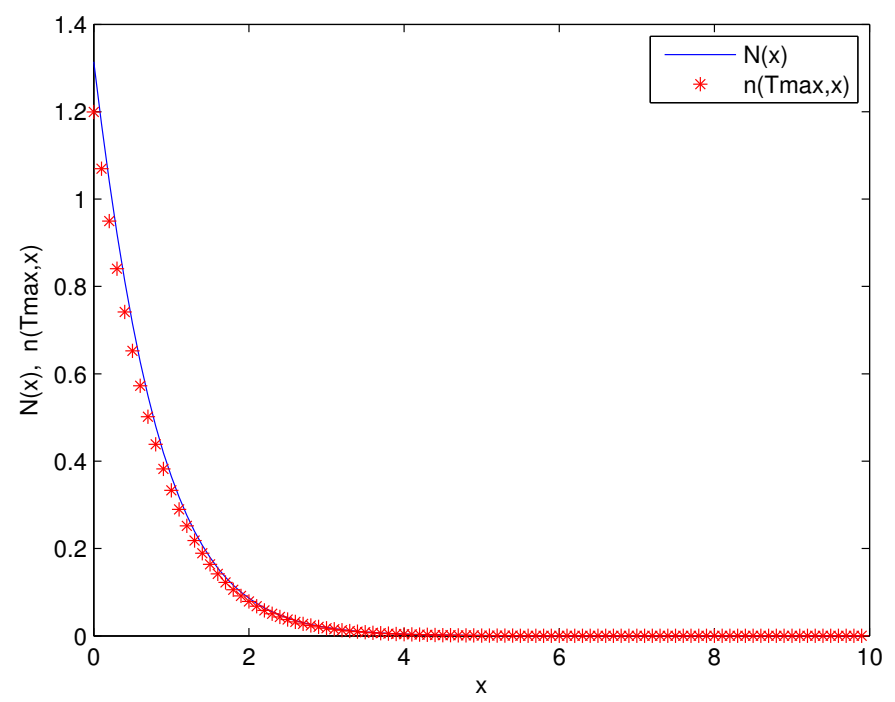

Figure 3. : $\operatorname{Tmax}=500$. Convergence of the solution to problem (1.2) towards the non trivial stationary solution of problem $(2.8)$

[4] W. A. Day, Extensions of property of solutions of heat equation to linear thermoelasticity and other theories, Quart. Appl. Math. 40, 1983, 319-330.

[5] O. Diekmann, M. Gyllenberg, H. Huang, M. Kirkilionis, J. A. J. Metz, H. R. Thieme, On the formulation and analysis of general deterministic structured population models. II. Nonlinear theory, J. Math. Biol., Vol 43, no. 2, 2001, 157-189.

[6] J.Z. Farkas and P. Hinow, Physiologically structured populations with diffusion and dynamic boundary conditions, Mathematical Biosciences and Engineering, Vol. 8, 2011, 503-513.

[7] M. Ianelli, M. Martcheva, F.A. Milner, Gender-structured Population Modeling: Mathematical Methods, Numerics, and Simulations, Frontiers in Applied Mathematics, SIAM, 2005, ISBN: 0898715776

[8] J. A. J. Metz, O. Diekmann, The dynamics of phisiologically structured populations. LN in biomathematics 68 , Springel-Verlag (1986)

[9] Michel P., General Relative Entropy in a nonlinear McKendrick model, AMS : Contemporary Mathematics 429, Stochastic Analysis and Partial Differential Equations (Gui-Qiang Chen, Elton Hsu, and Mark Pinsky), 205-232, 2007.

[10] P. Michel, S. Mishler, B. Perthame, General entropy equations for structured population models and scattering, C. R. Math. Acad. Sci. Paris, Vol. 338, (2004), 9, 697-702.

[11] P. Michel, S. Mishler, B. Perthame, General relative entropy inequality : an illustration on growth models, J. Math. Pures. Appl. Vol. 84, (2005), 9, 1235-1260.

[12] O. Kavian, Introduction à la théorie des points critiques, Springer-Verlag, 1993

[13] A. A. Kerefov, Non-local boundary value problems for parabolic equation, Differ. Ukravn. 15, 1979, 74-78.

[14] Y. H. Lee, L. Sherbakov, J. Taber, J. Shi, Bifurcation diagrams of population models with nonlinear diffusion, Journal of Computational and Applied Mathematics Vol. 194, (2006), 357-367.

[15] R. Dautray, J. L. Lions, Analyse Mathématique et calcul numérique pour les sciences et les techniques Masson, 1988.

[16] S. Mishler, B. Perthame, L. Ryzhik, Stability in a nonlinear population maturation model, Math. Model, Methods, Appl. Sci., Vol. 12, 1751-1772. 


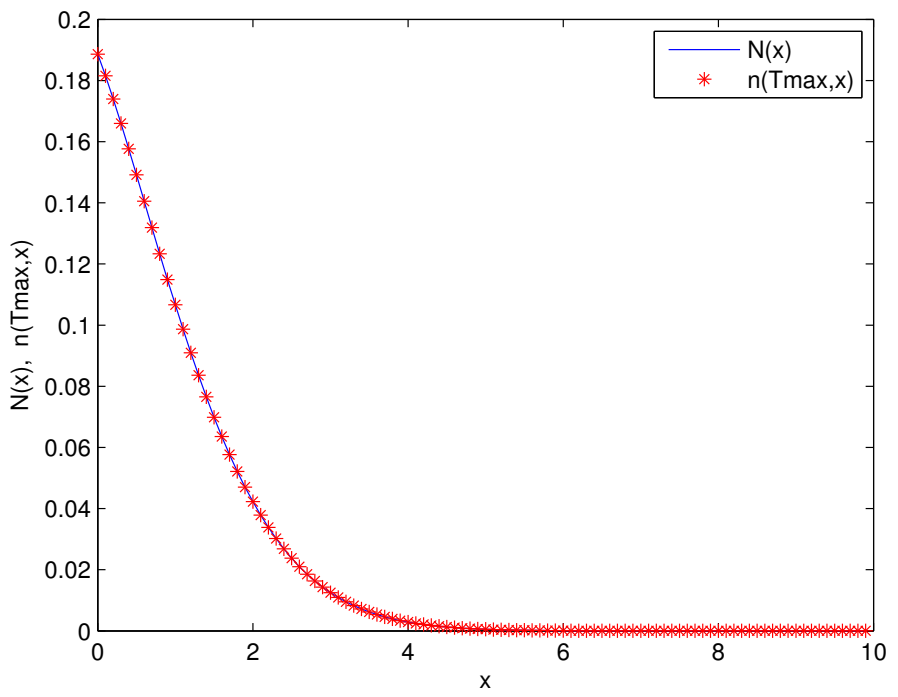

Figure 4. : $\operatorname{Tmax}=500, B(x)=x e^{-x}$, and $d(x)=x$. Convergence of the solution to problem (1.2) towards the non trivial stationary solution of problem $(2.8)$

[17] B. Opic, A. Kufner Hardy-type inequalities, Longman Scientific and Technical, New York, 1990.

[18] B. Perthame, L. Ryzhik, Exponential decay for the fragmentation or cell division equation. J. Diff. Eq. Vol. 210, (2005), 155-177.

[19] B. Perthame, Transport Equations in Biology, Birkhauster, Berlin, 2007.

[20] Vabishchevich, Non-local parabolic problems and the inverse heat-conduction problem, Differ. Ukravn. 17, 1981, 1193-1199.

[21] G.F. Webb, Theory of nonlinear age-dependent population dynamics, Monographs and Text- book in Pure and Applied Mathematics, Vol. 89, New-York Basel: Marcel Dekker Inc., 294p, 1985.

Tarik Mohammed Touaoula

Département de Mathématiques, Université Aboubekr Belkaïd, Tlemcen, Tlemcen 13000, Algeria.

E-mail address: tarik.touaoula@mail.univ-tlemcen.dz 